Provided for non-commercial research and education use. Not for reproduction, distribution or commercial use.

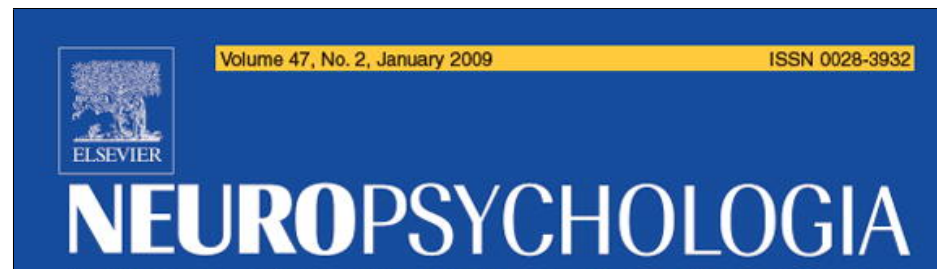

An international journal in behavioural and cognitive neuroscience

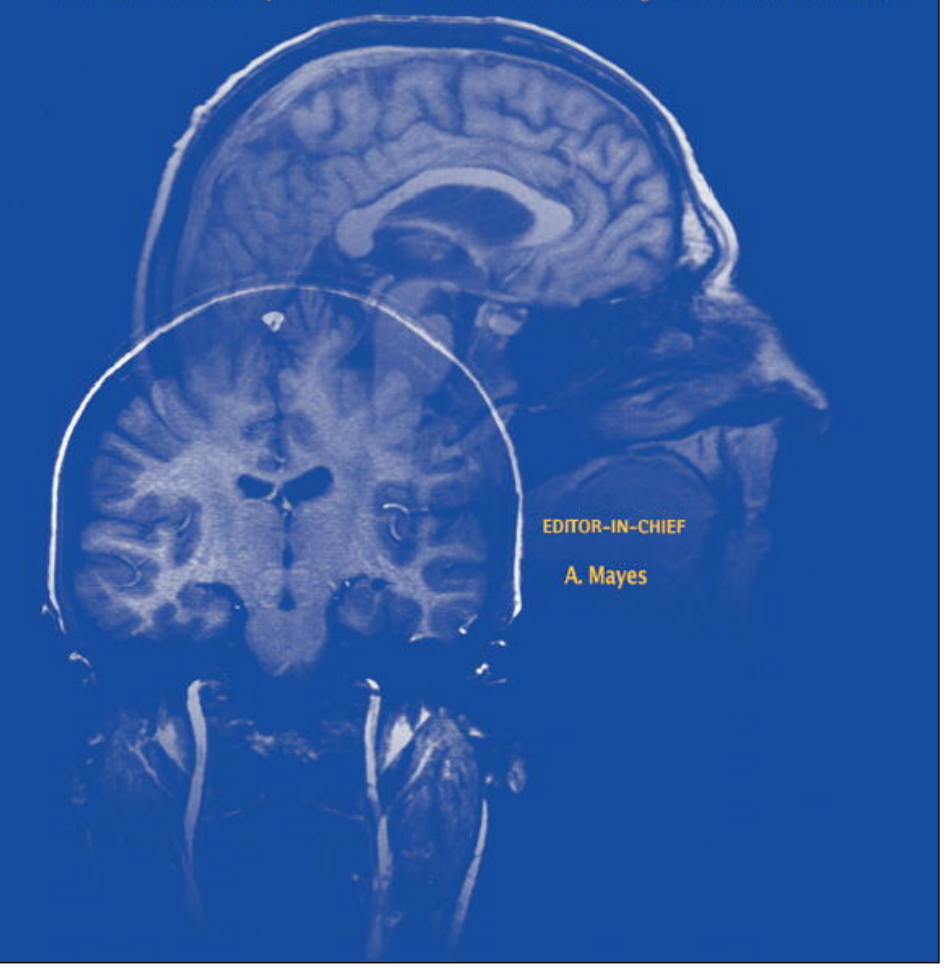

This article appeared in a journal published by Elsevier. The attached copy is furnished to the author for internal non-commercial research and education use, including for instruction at the authors institution and sharing with colleagues.

Other uses, including reproduction and distribution, or selling or licensing copies, or posting to personal, institutional or third party websites are prohibited.

In most cases authors are permitted to post their version of the article (e.g. in Word or Tex form) to their personal website or institutional repository. Authors requiring further information regarding Elsevier's archiving and manuscript policies are encouraged to visit:

http://www.elsevier.com/copyright 


\title{
Attentional orienting induced by arrows and eye-gaze compared with an endogenous cue
}

\author{
D. Brignani ${ }^{\mathrm{a}, \mathrm{b}}$, D. Guzzon ${ }^{\mathrm{b}}$, C.A. Marzi ${ }^{\mathrm{b}}$, C. Miniussi $^{\mathrm{c}, \mathrm{a}, *}$ \\ ${ }^{a}$ Cognitive Neuroscience Section, IRCCS Centro S. Giovanni di Dio Fatebenefratelli, Brescia, Italy \\ ${ }^{\mathrm{b}}$ Department of Neurological Sciences and Vision, University of Verona, Verona, Italy \\ c Department of Biomedical Sciences and Biotechnologies, National Neuroscience Institute, University of Brescia, Italy
}

\section{A R T I C L E I N F O}

\section{Article history:}

Received 27 January 2008

Received in revised form 26 August 2008

Accepted 8 September 2008

Available online 24 September 2008

\section{Keywords:}

Attention

Visual orienting

Automatic and voluntary orienting

Event-related potentials

ERPs

Central cues

\begin{abstract}
A B S T R A C T
Exogenous orienting has been widely studied by using peripheral cues whereas endogenous orienting has been studied with directional central cues. However, recent evidence has shown that centrally presented eye-gaze and arrows may produce an automatic rather than voluntary orienting of attention. Therefore, the aim of the present study was to investigate the behavioural and electrophysiological (event-related potentials-ERP) correlates of the attentional shift induced by arrows and eye-gaze. In order to have a control condition, we compared arrows and eye-gaze with a purely endogenous cue, i.e., a texture arbitrarily coding one direction. We analyzed the ERP components (P1, N1, P2a, P2p, P3) elicited by the cue stimuli and the early lateralised attentional effect (early directing attention negativity-EDAN). In addition, in order to investigate the topography of the neural mechanisms underlying the cortical activity in each cueing condition, we applied a temporal segmentation procedure. The results showed that the three cueing conditions induced a different strength of activation within the same cortical network. Occipito-parietal regions were involved in the early processing of visual information, followed by an involvement of frontal areas, likely implicated in learning associations. These data confirm the assumption that, in contrast to purely endogenous cues, arrows and eye-gaze induce a very fast attentional shift. However, the similarity of the ERP components and of the topographical cortical maps among conditions suggest that this early orienting of attention is more likely related to an overlearned association mechanism rather than to a real exogenous attentional process.
\end{abstract}

(c) 2008 Published by Elsevier Ltd.

\section{Introduction}

Two types of attentional orienting are traditionally described in the literature: endogenous orienting, that refers to the internal voluntary generated allocation of attention, and exogenous orienting, that refers to the automatic allocation of attention occurring in response to the sudden appearance of a peripheral visual event (Jonides, 1981). On the basis of behavioural, neuropsychological and physiological evidence, Corbetta and Shulman (2002) suggested that these types of attentional orienting are controlled by two partially segregated neural systems. One system is subserved by the dorsal posterior parietal and frontal cortex and is involved in the cognitive selection of sensory information and responses (goal-directed, endogenous attention); the second,

\footnotetext{
* Corresponding author at: Dipartimento di Scienze Biomediche e Biotecnologie, Sezione di Fisiologia, Università di Brescia, Viale Europa 11, 25123 Brescia, Italy. Tel.: +39 0303717441; fax: +390303717443.

E-mail address: miniussi@med.unibs.it (C. Miniussi).
}

which is largely lateralised to the right hemisphere and includes the temporoparietal and ventral frontal cortex, is recruited during the detection of behaviourally relevant stimuli, particularly when they are salient or unexpected (stimulus-driven, exogenous attention). However, neuroimaging studies that directly compared endogenous and exogenous orienting reported divergent results. On one hand, an overlap activation was found in a large fronto-parietal areas network, including bilateral premotor cortex, bilateral posterior parietal cortex and medial frontal cortex (Corbetta, Miezin, Shulman, \& Petersen, 1993; Nobre et al., 1997; Kim et al., 1999; Rosen et al., 1999). On the other, a rather wide range of areas showed differential activations between the two types of orienting: bilateral superior frontal cortex (Corbetta et al., 1993), left posterior parietal cortex (Nobre et al., 1997; Kim et al., 1999), bilateral temporo-occipital cortex (Kim et al., 1999) and right dorsolateral prefrontal cortex (Rosen et al., 1999). The discrepant results of these studies suggest that methodological factors (i.e., use of blocked design, type of exogenous orienting cues, adequate eye-movement control, use of proper random-effects analysis), may have influenced the neuroimaging results (Peelen, Heslenfeld, \& Theeuwes, 
2004). However, even when controlling for these factors, Peelen et al. (2004) found no difference in brain activation between endogenous and exogenous attentional orienting that activated the same fronto-parietal network.

In the light of these discrepant results, one possibility is that the difficulty to document neural differences between endogenous and exogenous attentional systems may be due to the fact that the central cues employed might have induced mixed orienting effects. Up to now, visual orienting has commonly been studied with paradigms in which cues correctly or incorrectly predict the location of a spatial target, producing faster (benefits) or slower (costs) responses, respectively. Centrally presented cues (typically an arrow) have been used to investigate endogenous orienting, while peripheral cues (luminance change) have been employed to study exogenous orienting (Posner, 1980; Lambert, Spencer, \& Mohindra, 1987; Muller \& Rabbitt, 1989; Yantis \& Jonides, 1984). For the past 20 years, central symbolic cues were thought to give rise only to endogenous orienting because they need to be interpreted to extract the positional information they convey (Jonides, 1981; Jonides \& Yantis, 1988; Muller \& Findlay, 1988; Muller \& Rabbitt, 1989; Yantis \& Jonides, 1984). However, recent behavioural findings have provided evidence that some kinds of centrally presented symbolic cues, such as eye-gaze and arrows, may produce automatic rather than voluntary orienting of attention. Importantly, this facilitatory effect occurs rapidly, as early as $100 \mathrm{~ms}$ after the appearance of the cue, even when cues are uninformative, that is they do not predict the location of the incoming target (Driver et al., 1999; Friesen \& Kingstone, 1998; Friesen, Ristic, \& Kingstone, 2004; Langton \& Bruce, 1999; Ristic, Friesen, \& Kingstone, 2002; Tipples, 2002). To date, this issue has been addressed mainly by means of behavioural studies, which provide a total measure of the whole sequence of processing stages (e.g., perception, attentional orienting, motor response).

In the present study we investigated the attentional shift induced by arrows and eye-gaze, by capitalising on the high temporal resolution of non-invasive electrophysiological recording methods. The relevance of event-related potentials (ERPs) for investigating spatial orienting has long been recognised (Eason, Harter, \& White, 1969; Hillyard \& Picton, 1979; Harter, Aine, \& Schroeder, 1982; Näätänen, 1975). However, a specific ERP marker able to disentangle endogenous from exogenous shift has not been identified yet. For this reason we compared ERPs elicited by arrows and eye-gaze with those elicited by a pure endogenous cue (i.e., two textures). The first two cues can be considered as benchmarks of directional cues while the textures have no intrinsic directional value and to trigger attentional orienting require a previously established texture-direction association.

We investigated the ERP components (P1, N1, P2a, P2p, P3) elicited by the three cues and the early attentional lateralised ERP component (early directing attention negativity-EDAN), which is assumed to reflect processes involved in the control of covert attentional shifts (Eimer \& Van Velzen, 2002; Eimer, van Velzen, \& Driver, 2002; Hopf \& Mangun, 2000; Nobre, Sebestyen, \& Miniussi, 2000; Yamaguchi, Tsuchiya, \& Kobayashi, 1994).

Hietanen, Leppanen, Nummenmaa, and Astikainen (2008) have recently reported a study with an aim similar to ours, in which they investigated ERPs elicited by non-predictive eye-gaze and arrows cues. They found an EDAN effect occurring with arrow but not with eye-gaze cues and other amplitude differences when the cueing stimuli were compared with neutral stimuli. These results were interpreted as providing evidence that eye-gaze and arrows are based on different neural mechanisms. However, the ERP pattern generated by eye-gaze and arrows was not compared with that generated by endogenous and/or exogenous cues, providing no indication about the nature of these mechanisms. In addition, the ERP analyses revealed only differences among conditions in the waveforms' amplitude but no information on whether the same or different neural networks were involved.

It is unanimously acknowledged that ERPs have an excellent temporal resolution but poor spatial resolution (Rugg \& Coles, 1995). However, the topographic analysis of ERPs may provide additional insights on how conditions differ in terms of likely underlying neurophysiologic mechanisms (Lehmann \& Skrandies, 1980; Michel, Seeck, \& Landis, 1999). Therefore, to disentangle the activation sequence of different cortical areas and to have important clues on the topography of the neural mechanisms underlying the execution of the tasks, we applied a temporal segmentation procedure (Murray, Brunet, \& Michel, 2008; Pascual-Marqui, Michel, \& Lehmann, 1995 for a tutorial review), a procedure which has been already applied in other cognitive domains (Itier \& Taylor, 2004; Pourtois, Dan, Grandjean, Sander, \& Vuilleumier, 2005, Pourtois, De Pretto, Hauert, \& Vuilleumier, 2006; Ruz \& Nobre, 2008).

In the present study we assumed that the cortical activation induced by textures reflects purely endogenous orienting mechanisms. According to the view that endogenous and exogenous orienting are subserved by different neural systems (Corbetta \& Shulman, 2002) one can predict that if arrows and eye-gaze produce an automatic orienting of attention, there should be an early difference in the cortical topography in comparison to textures. Also the EDAN effect is expected to be differently modulated by the type of attentional shift induced by the cues. EDAN has been linked to the encoding of the spatial information provided by cues and to the resulting beginning of the attentional shift (Harter, Miller, Price, LaLonde, \& Keyes, 1989; Hopf \& Mangun, 2000; Nobre et al., 2000). Therefore, differences in the amplitude or latency of EDAN across cueing conditions might witness an exogenous-like orienting of attention. In contrast, if arrows and eye-gaze activate an endogenous shift only, no topographical or EDAN differences should be observed in comparison to textures. In addition, the comparison between the pattern of cortical activation induced by arrows and eye-gaze should provide indications about the claim that gazetriggered attention is stronger and subserved by different brain systems than orienting to arrows (Friesen et al., 2004; Ristic et al., 2002).

Given that for the aim of the present experiment texture cues must be informative, we rendered all cues predictive of target location, thus maybe leading to attentional orienting on the basis of predictiveness. Endogenous and exogenous orienting are known to interact (Folk, Remington, \& Johnston, 1992; Leber \& Egeth, 2006), although it is still a matter of discussion whether top-down task set prevents or succeeds bottom-up phenomena (Eimer \& Kiss, 2008; Hickey, McDonald, \& Theeuwes, 2006). Nevertheless, since ERPs are able to detect the smallest differences among conditions, we still expected differences between cueing conditions at early stages of cortical activations. If arrows and eye-gaze induce an exogenous orienting, in the present task they should produce an additive process (exogenous plus endogenous) and therefore should show ERP differences in comparison to the exclusively endogenous textures.

\section{Methods}

2.1. Participants

A total of 24 neurologically normal paid volunteers ( 12 females), 19-38 years old ( mean $=25$ years), participated in the study. Twelve took part in the EEG Experiment 1 and twelve participated in a behavioural control Experiment 2. All participants were right-handed according to the Edinburgh handedness inventory test (+98.5\%) (Oldfield, 1971) and had normal or corrected to normal visual acuity. The study was approved by the local ethical committee and informed consent from participants was obtained prior to the beginning of the experiment. 


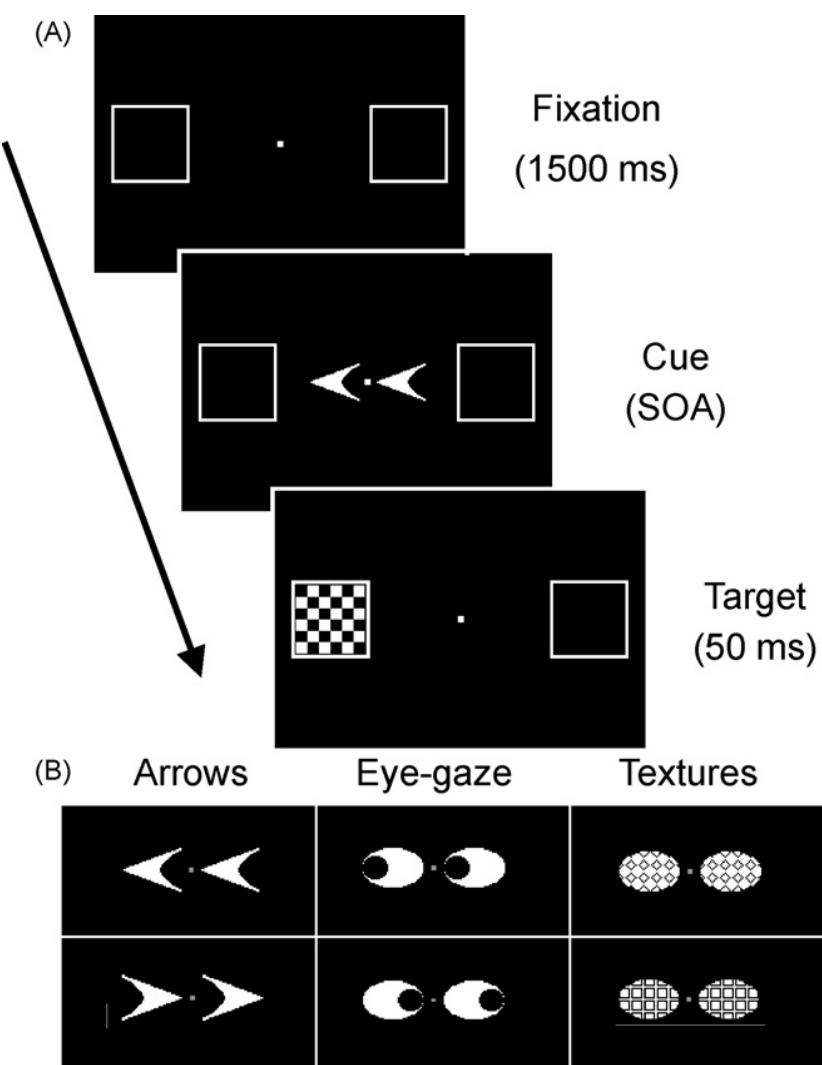

Fig. 1. Panel A: Schematic illustration of the behavioural task. Subjects fixated on a central point. A central cue appeared for a variable SOA $(200,700$ or $900 \mathrm{~ms}$ for Experiment 1 and 100, 200 or $700 \mathrm{~ms}$ for Experiment 2). At cue offset, a black and white checkerboard was flashed for $50 \mathrm{~ms}$ within one of the two peripheral boxes. Subjects had to detect its appearance by pressing as quickly as possible a button of the keyboard. The intertrial interval was $1500 \mathrm{~ms}$. Three types of cues, arrows, eyes and textures, were presented alternated in a blocked design. An example of valid trial with arrows cue is shown. Panel B: Illustration of the stimuli used as central cues: arrows, eyes, both directed either to the left or to the right, and two ellipses with different textures (rhombus and squares).

\subsection{Behavioural task and procedure}

The two experiments followed the same general procedure. The only difference was in the cue-target intervals. The task is schematically displayed in Fig. 1A. Stimuli were presented on a black background. The baseline display consisted of a small central fixation point and two outline boxes (squares of $2.82^{\circ}$ ) placed at $\pm 7.5^{\circ}$ eccentricity of visual angle along the horizontal meridian. At the beginning of each trial, the cue was superimposed on the fixation point for a variable interval of 200,700 or $900 \mathrm{~ms}$ (Experiment 1). At cue offset, a visual target was flashed for $50 \mathrm{~ms}$ within one of the two peripheral boxes. It consisted of a black and white checkerboard (same size as the boxes) with a spatial frequency of 2.15 cycles $/{ }^{\circ}$ of visual angle. Participants, while maintaining central fixation, had to detect the peripheral target and press as quickly as possible the spacebar of the keyboard. The intertrial interval was $1500 \mathrm{~ms}$.

Three types of cue were presented alternated in a blocked design session: two arrows, two schematic eyes and two textures (see Fig. 1B). Arrows and eyes could be both directed either to the right or to the left, while two different textures (squares and rhombus) were assigned a different directional meaning that was balanced across subjects: for half of the subjects the squares indicated the left and the rhombus the right while for the other half it was the opposite. All the cues were informative, that is, they correctly predicted the target location on $80 \%$ of the trials. Participants were informed on the probability of valid and invalid trials and were told to make use of this information to maximize their performance. The three cues had the same size ( $3.39^{\circ}$ width, $0.90^{\circ}$ height) and were designed in a way to minimize visual differences. Catch trials were intermingled to discourage from automatic responding. To the same purpose, the use of three stimulus onset intervals reduced the temporal predictability of the targets. The assignment of the response hand was balanced within and across participants: for each cueing condition (session) half of the participants responded with the left hand in the first four blocks and with the right hand in the last four, while it was the opposite for the other half of the participants.
Participants sat in a dimly illuminated room with the eyes at about $57 \mathrm{~cm}$ from a computer screen, and the experimenter ensured that they were centred with respect to the monitor and keyboard. They were instructed to maintain muscle relaxation and to avoid eye movements or blinks during task performance. Each cueing condition began with a training block.

The order of sessions, each one presenting only one type of cue, was counterbalanced across participants. Each cueing condition consisted of 452 trials divided into 8 small blocks ( 96 trials at 200 SOA; 160 trials at 700 SOA; 160 trials at 900 SOA 36 catch trials). Within each block, the condition (valid, invalid) and the SOA (200, $700,900)$ were presented in a random order. The entire experiment lasted about $2 \mathrm{~h}$, including short rest breaks after each block.

\subsection{ERP recordings and data processing}

The electroencephalographic (EEG) activity was recorded continuously from 27 sites using tin electrodes set in an elastic cap (Electro-Cap International, Inc.) and positioned according to the 10-20 International system (AEEGS, 1991). The montage included 3 midline sites (FZ, CZ, PZ), 12 sites over each hemisphere (F3/F4, F7/F8, FC1/FC2, FC5/FC6, C3/C4, T7/T8, CP1/CP2, CP5/CP6, P3/P4, P7/P8, PO7/PO8, 01/O2) and the right mastoid. Additional electrodes were used as ground and reference sites. The ground electrode was placed in front of FZ. The left mastoid served as reference for all electrodes. Data were recorded with a band-pass filter of DC- $100 \mathrm{~Hz}$ and digitized at a sampling rate of $500 \mathrm{~Hz}$ (SynAmps, NeuroScan). Electrode-skin impedance was set below $5 \mathrm{k} \Omega$. The electrooculogram (EOG) was recorded bipolarly through four additional electrodes placed on the left and right of the external canthi, for horizontal eye movements, and above and below the right eye, for blinks and vertical eye movements.

ERPs were constructed offline, according to stimulus type. First, EEG recordings were re-referenced to a weighted mean of both mastoid electrodes and re-filtered digitally with a band-pass of $0.01-45 \mathrm{~Hz}$. Epochs for the cue stimuli were constructed starting $200 \mathrm{~ms}$ before and ending $700 \mathrm{~ms}$ after cue presentation. Separate averages were computed for each type of cue (arrows, eye-gaze, textures) and for each cue direction (right, left). Only trials with long SOA were included (700 and $900 \mathrm{~ms}$ ) to avoid that cue- and target-related brain activity in short SOA $(200 \mathrm{~ms})$ could contaminate each other. All amplitude values were referred to the $200 \mathrm{~ms}$ pre-stimulus baseline. Epochs with behavioural errors, eye movements, blinks or muscle artefacts were excluded from analysis. Moreover, trials were automatically eliminated if the voltage in the epoch exceeded $\pm 100 \mu \mathrm{V}$ ( $18.9 \%$ of overall trials).

ERPs elicited by target stimuli were also analyzed in order to provide electrophysiological evidence of attentional orienting. In particular, we tested whether allocation of attention induced an enhancement of the early visual component P1 that has been previously shown to be modulated by spatial attention (Luck, Heinze, Mangun, \& Hillyard, 1990). Epochs were constructed starting $200 \mathrm{~ms}$ before and ending $300 \mathrm{~ms}$ after target presentation. Separate averages were performed for each type of cue (arrows, eye-gaze, textures), validity condition (valid, invalid) and cue direction (right, left). The rest of data processing was exactly the same as described for ERPs related to the cue presentation.

\subsection{Behavioural analysis}

In the behavioural analysis of Experiment 1, anticipations (RTs $<140 \mathrm{~ms}$ ), timedout trials (no response within $600 \mathrm{~ms}$ ), omissions and false-alarms on catch trials were classified as errors and excluded from analysis. Moreover, only trials with a good electrophysiological signal, that is, without blinks, ocular movements or muscular artefacts, were included in the behavioural analysis. This was done to assure a perfect correspondence between behavioural and electrophysiological data. A repeated-measures analyses of variance (ANOVA) was carried out on mean RT with Cue Type (eye-gaze, arrows, textures), SOA (200, 700, 900), Trial validity (valid, invalid) and Cue direction (right, left) as within-subject variables. In addition, in order to further investigate the validity effect, we performed an ANOVA on the differences in RTs between valid and invalid trials, including Cue Type (eye-gaze, arrows, textures), SOA $(200,700,900)$ and Cue direction (right, left) as factors. In both analyses, to assess the significant interactions, selected two-sample comparisons were performed by means of $t$-tests using the Bonferroni correction when appropriate. Only with textures a further analysis was performed to investigate possible learning effects. RTs were grouped according to trial block and submitted to an ANOVA with four factors: Block, SOA, Trial validity and Cue direction.

\subsection{ERP analysis}

The components of the ERPs elicited by cues were identified on the basis of their peaks as well as topographical analysis delineating successive stable functional states.

Conventional analyses were performed on the area of each component (P1, N1, P2a, P2p, P3) within temporal windows centred on the peak latency of the components. In addition, the mean amplitude obtained within specific latency ranges was analyzed to reveal the attentional lateralised ERP component (EDAN). The electrodes included in each analysis and the latency ranges are detailed in Section 3. Separate analyses were carried out for each component with repeated-measures 
Table 1

Mean reaction times for the different experimental conditions in the ERP experiment 1 .

\begin{tabular}{llllll}
\hline Validity & Cue type & \multicolumn{2}{l}{ SOA (ms) } & & \\
\cline { 3 - 6 } & & 200 & 700 & 900 & Mean \\
\hline \multirow{3}{*}{ Valid } & Arrows & 307 & 261 & 256 & 275 \\
& Eye-gaze & 308 & 263 & 258 & 276 \\
& Textures & 332 & 260 & 255 & 282 \\
& Arrows & 337 & 311 & 310 & 320 \\
\multirow{4}{*}{ Invalid } & Eye-gaze & 325 & 315 & 304 & 315 \\
& Textures & 351 & 334 & 325 & 337 \\
& Valid & 316 & 261 & 256 & 278 \\
& Invalid & 338 & 320 & 313 & 324 \\
\hline
\end{tabular}

ANOVAs including the factors: Cue type (arrows, eye-gaze, textures), Cue direction (right, left), Hemisphere (right, left) and Electrode site for ERPs locked to the cue stimuli.

The P1 component elicited by target stimuli was analyzed with a repeatedmeasures ANOVA considering the factors: Cue type (arrows, eye-gaze, textures), Trial validity (valid, invalid), Hemisphere (contralateral, ipsilateral to the target side) and Electrode site. For all ERP analyses, the Greenhouse-Geisser epsilon correction factor was applied to compensate for possible effects of non-sphericity in the measurements. Only the corrected probability (as well as epsilon) values are reported.

To determine whether the three cuing conditions were associated with distinct topographies of neural activity, we applied a temporal segmentation procedure (Pascual-Marqui et al., 1995), using CARTOOL software (version 3.32; developed by Brunet, Functional Brain Mapping Laboratory, Geneva, Switzerland). This procedure identifies a sequence of statistically distinct topographic maps, called functional microstates, that are thought to correspond to steps in information processing during which a brain region or network remain active. Changes in the topography across different conditions reflect an activation of distinct neural generators and may arise independently of differences in strength (i.e., amplitude of the component waveforms) (Lehmann \& Skrandies, 1980). Thus, such analysis can provide important additional information about spatio-temporal dynamics of visual processing, not always available in conventional waveform measures (Michel et al., 1999). We compared topographies elicited by each type of cue starting from cue onset until $700 \mathrm{~ms}$ (175 time frames). The analysis was carried out using the Atomize \& Agglomerate Hierarchical Clustering approach. The optimal number of topographic maps and their times of occurrence were selected on the basis of a cross-validation criterion in which the smallest number of segment maps explaining the greatest amount of variance was retained (Pascual-Marqui et al., 1995). Results revealed that the group-averaged data could be segmented by the same solution of six different maps, explaining $94 \%$ of the total variance. To statistically compare how well the topographies explained variance across participants and conditions, for each map we measured the amount of variance (GEV) and the degree of expression (in time frames TFs) in every participant and condition, using a spatial fitting procedure (Michel et al., 1999). In order to assess if maps better explained a specific condition, we entered the fitting values of each map in an ANOVA considering Cue type (arrows, eye-gaze, textures) as factor.

\section{Results}

\subsection{Behavioural data}

In Experiment 1, 0.6\% and $0.2 \%$ of the trials were excluded from the analysis, respectively for anticipations and timed-out. Participants performed the task with high levels of accuracy (mean accuracy $=97.6 \%$; range $=95.1-99.7 \%$ ). False-alarms occurred on $1 \%$ and omissions on $1.4 \%$.

Statistical analysis revealed a significant main effect of Trial validity $[F(1,11)=51.72, p<0.001]$, with faster responses for valid than invalid trials (see Table 1 ). The factor Trial validity interacted with $\operatorname{SOA}[F(2,22)=29.34, p<0.001]$, indicating that the validity effect, although significant at all SOAs, was significantly larger at SOA-700 $(p<0.001)$ and SOA-900 $(p<0.001)$ with respect to SOA200. There was no difference in the validity effect between SOA-700 and SOA-900 $(p=1)$. Trial validity interacted also with Cue type and $\operatorname{SOA}[F(4,44)=5.27, p=0.001]$ although a significant difference between valid and invalid trials was found with all cues at all SOAs (p's $<0.02$ ). The analysis on the differences in RTs showed that with
Table 2

Mean reaction times for the different experimental conditions in the control behavioural Experiment 2.

\begin{tabular}{llllll}
\hline Validity & Cue type & \multicolumn{2}{l}{ SOA $(\mathrm{ms})$} & & \\
\cline { 3 - 6 } & & 100 & 200 & 700 & Mean \\
\hline \multirow{3}{*}{ Valid } & Arrows & 304 & 296 & 285 & 295 \\
& Eye-gaze & 315 & 305 & 293 & 304 \\
& Textures & 341 & 325 & 291 & 319 \\
& Arrows & 327 & 329 & 336 & 331 \\
\multirow{3}{*}{ Invalid } & Eye-gaze & 344 & 341 & 339 & 341 \\
& Textures & 345 & 337 & 342 & 341 \\
& Valid & 320 & 309 & 290 & 306 \\
& Invalid & 339 & 336 & 339 & 338 \\
\hline
\end{tabular}

all the cue types the validity effect was significantly larger at longer SOAs. More importantly, at SOA-200 there was no difference in the validity effect between the cues, even if a trend was observed for arrows to have a larger validity effect in comparison to eye-gaze $(p=0.053)$ and textures $(p=0.08)$. No difference in the validity effect between cues was found either at SOA-700 or at SOA-900, although a trend emerged at SOA-700 for textures to have a larger validity effect in comparison to eye-gaze $(p=0.06)$ and textures $(p=0.06)$.

In the main ANOVA there was also a significant main effect of $\operatorname{SOA}[F(2,22)=30.57, p<0.001]$ with longer RTs at the shortest SOA (200 ms), reflecting a typical cue-target foreperiod effect. No difference was found between SOA-700 and SOA-900. There was also a significant effect of the main factor Cue type $[F(2,22)=5.7$, $p=0.01]$ and a significant two-way interaction Cue type by $\operatorname{SOA}[F(4$, $44)=6.03, p<0.001]$, indicating faster responses when targets were preceded by eye-gaze or arrows than when they were preceded by textures and this difference was particularly evident at SOA-200. Finally, no significant effect related to the factor Block emerged in the analysis ruling out learning effects in the texture condition (i.e., no increase of validity effect in the last block compared to the first one).

Since the behavioural results provided no conclusive evidence about the shortest SOA necessary for the cues to induce spatial orienting, a successive behavioural experiment was carried out to investigate cueing effects before $200 \mathrm{~ms}$. The task was exactly the same as in Experiment 1 except for the cue-target SOA which was 100, 200 and $700 \mathrm{~ms}$. Participants performed in a balanced order the three cueing conditions, each consisting of 390 trials divided in 6 blocks ( 120 trials for each SOA and 30 catch trials). ANOVAs testing the same factors as in Experiment 1 were performed.

$0.6 \%$ and $0.5 \%$ of the trials were excluded from the analysis, respectively for anticipations and timed-out. As in the preceding experiment, accuracy of performance was very high (mean accuracy $=98.3 \%$; range $=96-99.5 \%$; false-alarms: $0.8 \%$, omissions: $0.9 \%)$. Most of the results were consistent with the previous experiment.

A main effect of Trial validity $[F(1,11)=44.70, p<0.001]$ was found and it interacted with $\operatorname{SOA}[F(2,22)=30.75, p<0.001]$, revealing a significant larger validity effect at SOA-700 as compared to the shorter SOAs ( $p$ 's $<0.001$ ) (see Table 2). The factor Trial validity interacted with Cue Type $[F(2,22)=4.97, p=0.02]$, showing that the validity effect, although significant, was significantly smaller with textures in comparison to eye-gaze $(p=0.03)$ and arrows $(p=0.05)$. Interestingly, the factor Trial validity interacted also with Cue type and $\operatorname{SOA}[F(4,44)=2.53, p=0.05]$ and subsequent analyses revealed that with arrows and eye-gaze a significant difference between valid and invalid trials was found at all SOAs ( $p$ 's $<0.005)$. Importantly, with texture cues the validity effect emerged only at SOA-700 $(p=0.001)$ while a trend was observed with SOA-200 $(p=0.06)$ and no difference between valid and invalid trials was 

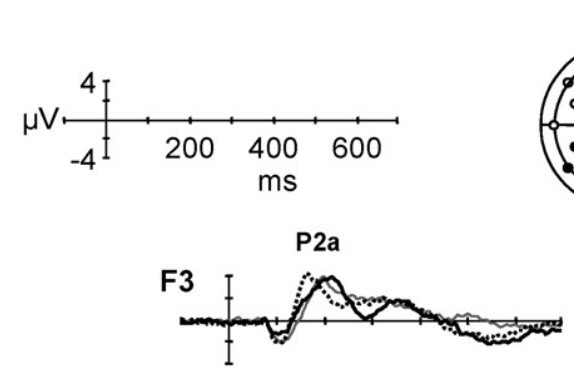

C3

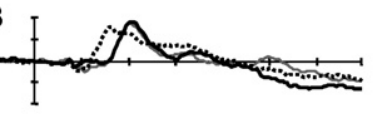

CP5

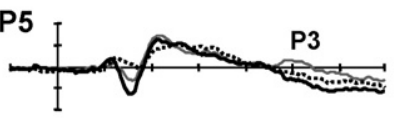

P3

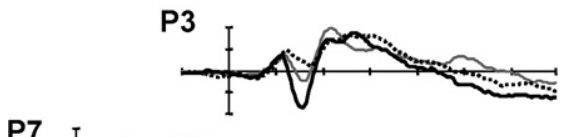

P7
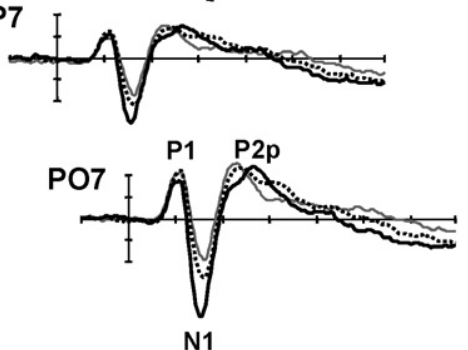

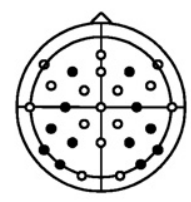

F4

HEOG

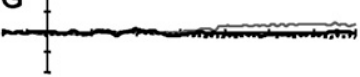

VEOG

P2a
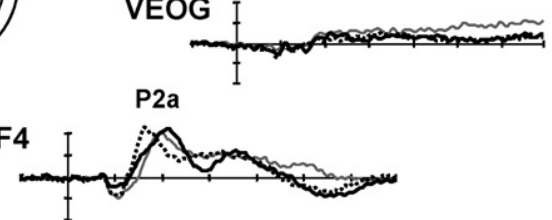

C4

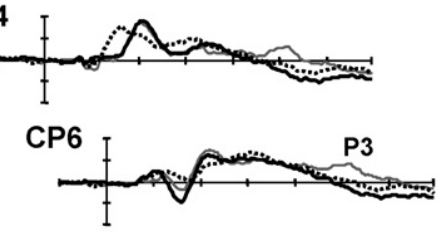

P4

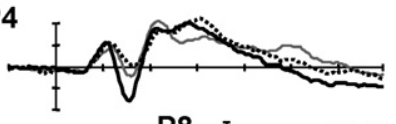

P8

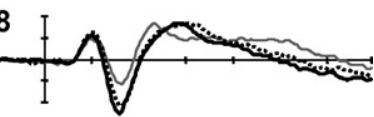

Arrows

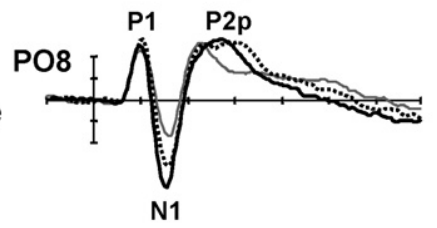

Fig. 2. Grand average ERPs $(N=12)$ elicited by the three central cues at central and parietal sites where most of the components were analyzed. The electrode montage is shown with the displayed sites shaded black. Voltage is plotted with positive values upward.

found at SOA-100 $(p=0.4)$. Thus, participants were not able to take advantage of the information provided by textures with a cue-target interval of $100 \mathrm{~ms}$. Importantly, there was no difference in the validity effect between arrows and eye-gaze at any SOAs ( $p$ 's $>0.4)$. The validity effect of textures was significantly smaller in comparison with arrows and eye-gaze both at SOA-100 and SOA-200 ( $p$ 's < 0.04), but not at SOA-700.

The main ANOVA revealed also a main effect of $\operatorname{SOA}[F(2$, $22)=6.10, p=0.008]$ and a SOA by Cue type interaction $[F(4$, $44)=3.74, p=0.01$ ], showing that RTs were faster at the longest SOA $(700 \mathrm{~ms})$ with respect to the shortest one $(100 \mathrm{~ms})$ when targets were preceded by eye-gaze or textures.

\subsection{ERP data}

\subsubsection{Cues}

Fig. 2 shows ERPs in response to the three central cues. As can be seen, each type of cue elicited the early visual components P1, $\mathrm{N} 1$, a frontal and a posterior P2, a late $\mathrm{P} 3$ and a slow negative wave linked to response preparation.

The significant effect of the main factor Cue type emerged in the analysis of P1 and $\mathrm{N} 1$ revealing that the area of these early visual components was modulated by the type of cue.

P1 (measured at PO7/8, 01/2; latency range $80-130 \mathrm{~ms}$ ) showed a significantly smaller area with arrows than with eye-gaze $(p=0.001)$ and textures $(p<0.001)$, while no difference emerged between the last two [Cue type $F(2,22)=12.75, p<0.001, \varepsilon=0.991$ ]

$\mathrm{N} 1$ (measured at $\mathrm{P} 7 / 8, \mathrm{PO} / 8$; latency range $130-180 \mathrm{~ms}$ ) showed a highly significant Cue type effect $[F(2,22)=42.25$, $p<0.001, \varepsilon=0.905]$ with a different area for all the three cues (arrows vs. eye-gaze $p=0.001$; arrows vs. textures $p<0.001$; eye- gaze vs. textures $p<0.001)$. The arrows induced the largest $\mathrm{N} 1$ $\left(-290.4 \mu \mathrm{V}^{2}\right)$ followed by eye-gaze $\left(-208.2 \mu \mathrm{V}^{2}\right)$ and textures $\left(-102.2 \mu \mathrm{V}^{2}\right)$. Moreover, a significant Cue type by Electrode site interaction $[F(2,22)=9.88, p<0.001, \varepsilon=0.917]$ revealed that this difference was largest at the $\mathrm{PO} / 8$ electrodes.

The analyses of both the frontal and the parietal P2 components (measured, respectively: P2a at F3/4, F7/8, FC1/2, FC5/6; latency range centred on the peak latency: eye-gaze: $150-200 \mathrm{~ms}$; arrows and textures: 170-220 ms; P2p at P3/4, P7/8, PO7/8; latency range $200-300 \mathrm{~ms}$ ) revealed no main effect or interactions of the Cue type factor.

Finally, a late P3 component (measured at CP1/2, CP5/6, P3/4, $\mathrm{P} 7 / 8$; latency range $460-560 \mathrm{~ms}$ ) was more pronounced in the texture than in the arrow and eye-gaze cueing conditions, according to the significant main factor Cue type $[F(2,22)=10.84, p=0.003$, $\varepsilon=0.652]$. This component reached also the largest area in the right in comparison to the left hemisphere (Hemisphere $[F(1,11)=7.57$, $p=0.02, \varepsilon=1.000]$ ).

With regard to the attentional lateralised ERP component, the presence of lateralised effects sensitive to the direction of an attentional shift should be reflected by the Cue Direction by Hemisphere interaction (Eimer \& Van Velzen, 2002; Eimer et al., 2002). No EDAN was found over the posterior electrodes CP5/6, P3/4, P7/8 and $\mathrm{PO} 7 / 8$ in the conventional temporal interval reported in literature (from $\sim 150$ to $400 \mathrm{~ms}$ ), but a similar effect was found over the same electrodes during an earlier time window between 100 and $170 \mathrm{~ms}$ (Fig. 3). Interestingly, this EDAN-like effect did not concern the three cues in the same way, as revealed by the significant three-way interaction Cue type by Cue direction by Hemisphere $[F(2$, $22)=4.65, p=0.02, \varepsilon=0.812$ ]. Subsequent analyses conducted separately for each cue revealed that only arrows showed the Cue 
(A)
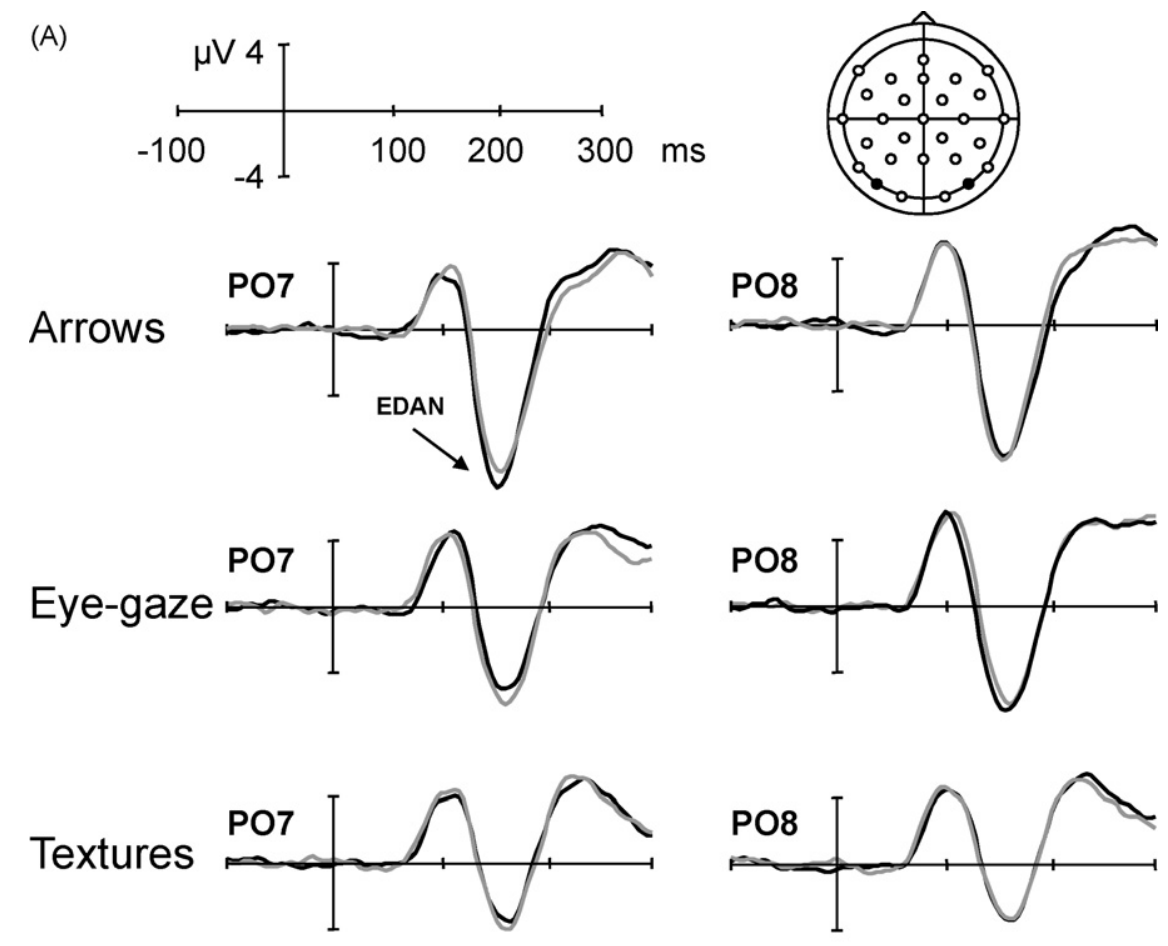

(B)

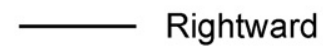

Leftward

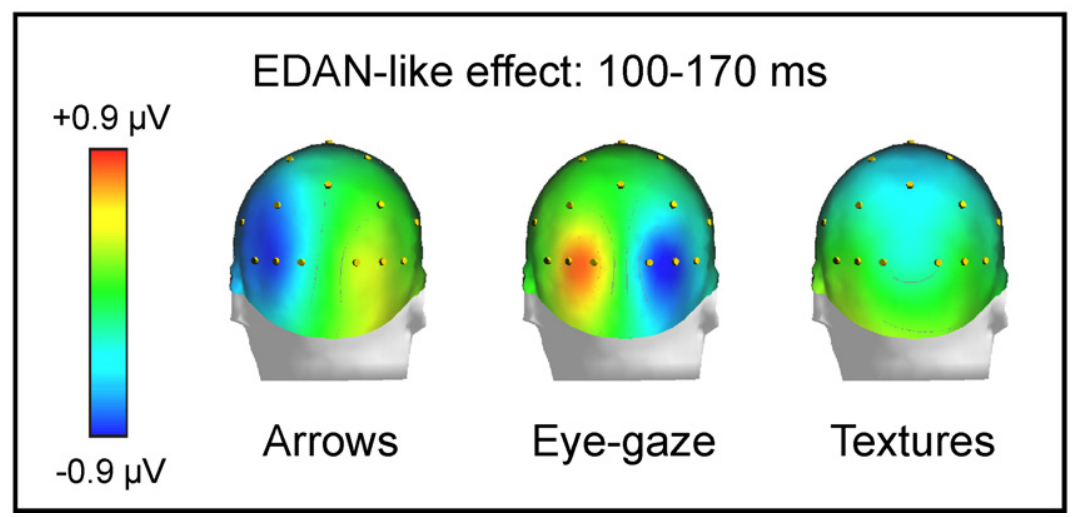

Fig. 3. Panel A: The grand average ERPs $(N=12)$ elicited by rightward (in black) and leftward (in grey) cues over the parietal PO7/8 electrodes is shown for each type of central cue. In PO7 it can be seen that only arrows toward the right elicited a larger negative response respect to arrows toward the left. An inverse pattern is shown by eye-gaze. Panel B: Scalp topographies of the EDAN-like effect for each type of cue (ASA software, A.N.T.). The maps plot the different waveforms between 100 and 170 ms, obtained by subtracting ERPs in response to cues directing attention to the left from ERPs in response to cues directing attention to the right. Enlarged negativities related to rightward attentional orienting are reflected by blue colour, while enlarged negativities related to leftward attentional shift are reflected by red colour.

Direction by Hemisphere interaction $[F(1,11)=6.48, p=0.02, \varepsilon=1]$, indicating that only over the left posterior hemisphere arrows pointing to the right elicited a larger negative response with respect to arrows pointing to the left. In the eye-gaze analysis a lateralised effect limited to the electrode site PO7 emerged $[F(3,33)=5.18$, $p=0.03, \varepsilon=0.406$ ], but showed an inverse pattern in comparison with arrows: eye-gaze toward the right induced a smaller negative response with respect to eye-gaze toward the left. Finally, the textures showed no effect sensitive to the direction of the attentional shift.

The segmentation procedure revealed that, starting from about $70 \mathrm{~ms}$ after cue presentation, the three cueing conditions were described by the same succession of six topographical maps (see Fig. 4). Maps 1, 2, 3 and 4 corresponded, respectively to the P1, N1, P2a and P2p components. Maps 1 and 4 showed a high correlation index between the respective clusters of activity, as well as maps 2 and 3. Statistical analyses showed no main effect of cue type suggesting that the temporal appearance and the amount of variance explained by the maps were identical among the three cueing conditions.

\subsubsection{Targets}

Consistent with prior reports, there was a significant main effect of Trial Validity $[F(1,11)=15.48, p=0.002]$ (Fig. 5) with a larger amplitude of the posterior P1 (measured at P7/8 and PO7/8; latency range $105-145 \mathrm{~ms}$ ) for valid than invalid trials. The factor Trial Validity interacted with Hemisphere $[F(1,11)=5.66, p=0.04]$ and with Hemisphere and Electrode site $[F(1,11)=4.68, p=0.05]$, showing that the validity effect was present in both the electrodes of the ipsilateral hemisphere, but only in the $\mathrm{P} 7 / 8$ electrodes of the contralateral hemisphere. 


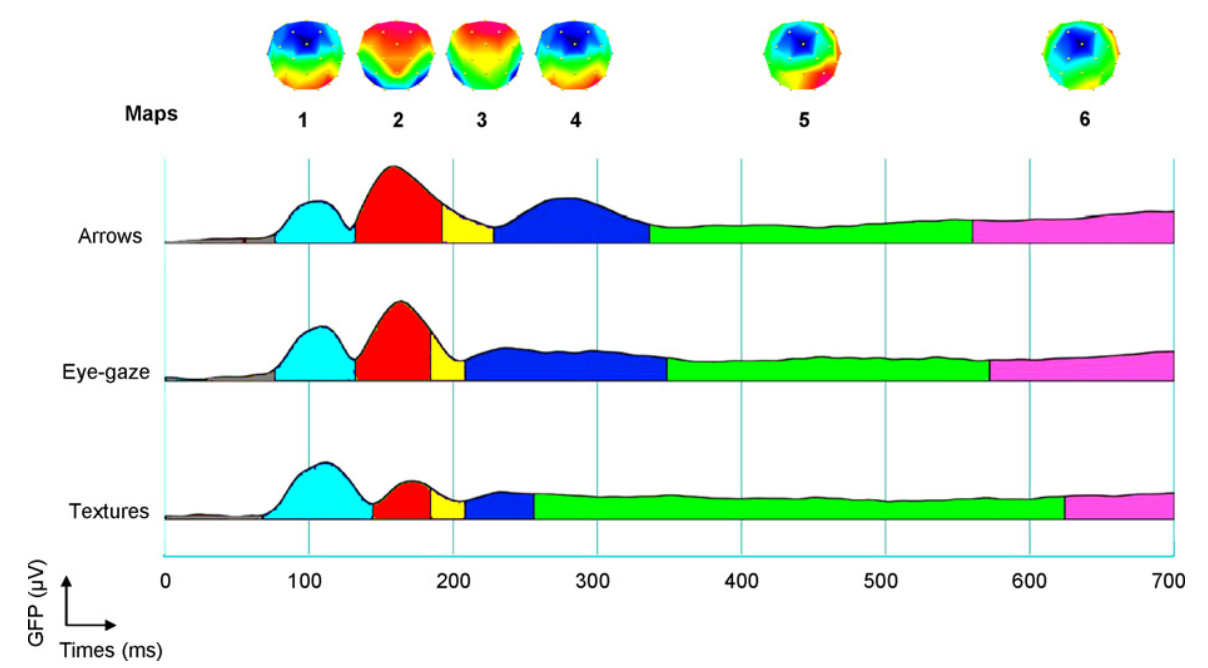

Fig. 4. The results of the temporal segmentation procedure performed on grand averages of all conditions. The analysis (from cue onset until $700 \mathrm{ms)} \mathrm{showed} \mathrm{a} \mathrm{segmentation}$ solution with six different clusters of activity (or topographical maps), indicated in the figure as coloured segments. The succession of these maps is shown for arrows, eye-gaze and texture. Time is represented along the $x$-axis and global field power (GFP) along the $y$-axis. Different colours are used simply to indicate distinct topographies. Axial views of the electric field configuration (scaled to unitary strength by dividing the voltage at each electrode by the GFP) are shown at the top of the figure, each one corresponding to one numbered map segment.

In addition, a significant effect of the main factor Cue type emerged $[F(2,22)=7.44, p=0.01]$, indicating that P1 was larger when the target was preceded by arrows than by textures. The Cue type factor did not interact with any other factor.

\section{Discussion}

In this study we investigated the behavioural and electrophysiological correlates of attentional orienting induced by arrows and eye-gaze in comparison with those induced by a pure endogenous cue (textures). We expected that the analysis of the ERP components together with a topographic analysis could provide relevant information about the nature of the attentional shift elicited by these cues. The results of the behavioural analysis confirmed the general efficacy of the cues: participants were faster to respond when targets appeared at the cued with respect to the uncued location. This validity effect (mean: $46 \mathrm{~ms}$ ) was present with all types of cue at all SOAs. As expected, both arrows and eye-gaze induced early attentional effects, but surprisingly, also textures did, despite they required retrieval of the previously established texture-direction association. Furthermore, we carried out another behavioural experiment in order to investigate cueing effects earlier than SOA 200. Participants performed the same task as in Experiment 1 with an additional shorter SOA $(100 \mathrm{~ms})$. As expected, at SOA100 participants were still able to take advantage of the information provided by arrows and eye-gaze, but not of that provided by textures. Thus, the present behavioural data support our choice of considering textures as a purely endogenous cue given that at a very short SOA they yielded different effects in comparison to directional symbolic cues. The cue-target interval of $200 \mathrm{~ms}$ appeared to be borderline for textures to trigger an attentional shift. Traditionally, behavioural effects of spatial orienting based on symbolic cues were thought to take place with cue-target intervals of 200-300 ms (Gibson \& Bryant, 2005; Warner, Juola, \& Koshino, 1990). Since textures had no intrinsic directional meaning, their ability to trigger an attentional shift was expected to take more time. However, previous evidence suggested that topdown control settings may influence attentional orienting earlier

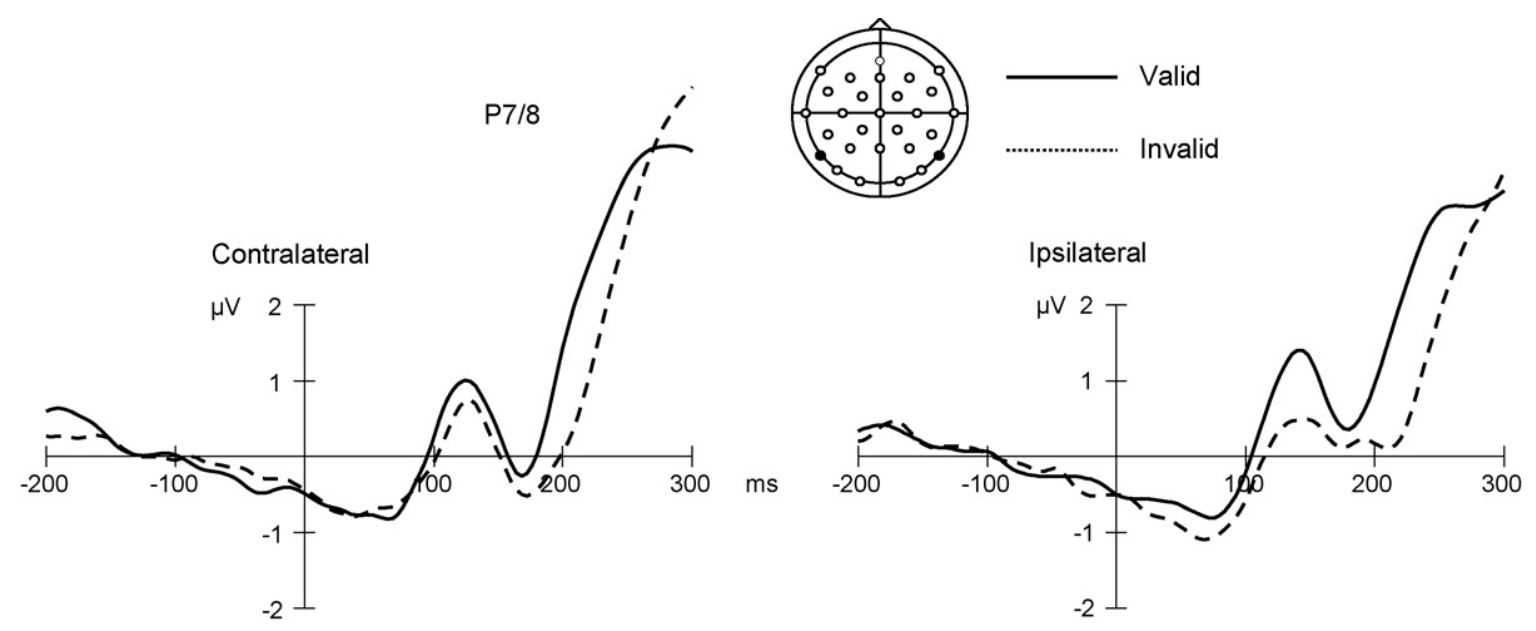

Fig. 5. Grand average ERPs $(N=12)$ elicited by target stimuli at P7 and P8 electrodes for valid (solid line) and invalid (dotted line) trials. The electrode montage is shown with the displayed sites shaded black. Voltage is plotted with positive values upward. 
and more profoundly than conventionally thought (Folk et al., 1992).

Experiment 1 and Experiment 2 differed with regard to the SOAs employed and also to the distribution of trials across SOAs. In the former there were more trials at long SOAs while in the latter the trials were equally distributed across SOAs. Previous studies which investigated exogenous spatial cueing effects with peripheral non-predictive cues showed that the specific SOAs used (Cheal \& Chastain, 2002) and their distribution (Gabay \& Henik, 2008; Milliken, Lupianez, Roberts, \& Stevanovski, 2003) engendered temporal expectations influencing attentional effects. However, the same studies demonstrated that temporal expectancy affected the attentional set only in discrimination tasks (Milliken et al., 2003), while this was not the case in simple tasks not requiring deep processing of the target (Gabay \& Henik, 2008) such as in the present experiments. These remarks make it unlikely that the different distribution of trials across SOAs in the two experiments influenced attentional orienting effects.

Finally, another behavioural result which deserves to be highlighted is that arrows and eye-gaze induced the same attentional effects with no difference either in the strength or in the timecourse of the cueing effects. Although gaze-triggered attention has been proposed to represent a special and unique type of reflexive orienting (Friesen \& Kingstone, 1998; Friesen et al., 2004), an increasing number of studies have shown that also arrows induce automatic-like shifts of attention (Gibson \& Bryant, 2005; Hommel, Pratt, Colzato, \& Godijn, 2001; Ristic et al., 2002; Tipples, 2002).

The aim of the present ERP experiment was to find out whether these identical behavioural effects were subserved by the same or by distinct neural attentional mechanisms. Previous ERP experiments of spatial attention triggered by eye-gaze focused mainly on ERPs elicited by peripheral targets following eye-gaze cues (Schuller \& Rossion, 2001, 2004). They demonstrated that the early visual components P1 and N1 were enhanced when they were elicited by targets presented at congruent versus incongruent gazed-at locations. These amplitude modulations were interpreted as evidence that eye-gaze induces an attentional shift and a subsequent amplification of early sensory processing. In the present experiment we used a different strategy (see also Eimer \& Van Velzen, 2002; Harter et al., 1989; Hopf \& Mangun, 2000; Nobre et al., 2000; Talsma, Slagter, Nieuwenhuis, Hage, \& Kok, 2005; Yamaguchi et al., 1994) and compared ERPs triggered by different cues assuming that they would reflect correspondingly different mechanisms of covert shifts of spatial attention.

Topographical segmentation of ERPs elicited by the three cueing conditions separated the waveforms into an identical succession of topographical maps. Since the presence across conditions of maps, having the same identification number and overlapping in time represents the same cluster of activity (Pascual-Marqui et al., 1995), these results pointed toward the same stages of the neural mechanisms engaged by the three cueing conditions. The first map described a posterior positivity, corresponding to the visual P1, as well as a concurrent frontal negativity. This topography represented the visual processing of the central cues and showed no difference among the three cueing conditions, except for the strength of the activation. Indeed, arrows generated a smaller P1 with respect to eye-gaze and textures. Even though modulations of P1 amplitude have been shown to be related to spatial attention (Hopfinger \& Mangun, 1998, 2001; Mangun \& Hillyard, 1991; Luck et al., 1994), the present amplitude difference is more likely to result from the slight physical differences between the cues. Despite the attempt to minimize these differences, arrows had a different shape with respect to eye-gaze and textures, which were both oval. This physical difference is probably sufficient to explain the P1 amplitude modulation.
The second and the third map showed a posterior negativity, corresponding to N1, followed by a frontal positivity (P2a). Again, the difference between the cueing conditions in the amplitude of the waveforms but not in their topography, suggests a different activation of the same neural network. Many studies of visualspatial attention have found that the $\mathrm{N} 1$ amplitude is modulated by attentional manipulations and several functional interpretations have been proposed (Luck et al., 1990; Mangun \& Hillyard, 1991; Vogel \& Luck, 2000). The N1 has proven to be especially sensitive to operations of discrimination within the focus of attention (Arnott, Pratt, Shore, \& Alain, 2001; Vogel \& Luck, 2000). The N1 modulations observed in the present study represent more likely differences in the attentional processing among cueing conditions and this is supported also by the presence of a frontal positive component (P2a). Several studies have described a prefrontal positivity, termed anterior P2 (Potts \& Tucker, 2001; Potts, 2004; Potts, Patel, \& Azzam, 2004), frontal P3 (Makeig et al., 1999) or frontal selection positivity (Anllo-Vento, Luck, \& Hillyard, 1998; Kenemans, Kok, \& Smulders, 1993), occurring at about the same latency as the posterior negativity. Although frontal positivity and posterior negativity have been hypothesized to represent opposite poles of the same neural generator, differences in latency (Wijers, Mulder, Okita, \& Mulder, 1989), in psychological responsiveness (Potts, Liotti, Tucker, \& Posner, 1996) and in laterality (Potts, 2004) have suggested distinct neural substrates. N1 indexes activity in specific regions of ventral and lateral extrastriate visual cortex (Clark \& Hillyard, 1996; Gomez Gonzales, Clark, Fan, Luck, \& Hillyard, 1994; Mangun et al., 1993), while the neural generators of the P2a are thought to be located in orbitofrontal cortex (Potts et al., 2004). These two components reflect separable cognitive operations: the posterior negativity has been linked to stimulus specific processes enhanced by attention, while the frontal positivities have been interpreted as indexing operations related to the task-relevance of stimulus items (Kenemans et al., 1993; Potts et al., 2004; Smid, Jakob, \& Heinze, 1999). In keeping with these studies, the differences in N1 amplitude seen here could indicate the allocation of attention to task-relevant features. Since participants were instructed to shift their attention as quickly as possible to the side indicated by the cues it is possible that they used primitive features of the cue stimuli to infer the direction indicated by the cue. Thus, the early enhancement of the $\mathrm{N} 1$ could arise from the allocation of attention based on these primitives, even prior to full classification or recognition of the stimuli. As shown in previous studies, this effect could be based on an attentional set established as top-down bias prior to stimulus delivery (Driver \& Frackowiak, 2001; Potts et al., 2004). In arrow and eye-gaze cues, the high-luminance regions appeared to be the first feature useful to drive attention. This features, respectively ipsi- and contra-laterally to the direction of attention, could have been detected more promptly with arrows than with schematic eyes, explaining the larger N1 with arrows. Finally, the higher complexity of texture cues could instead account for the smallest N1.

The fourth and the fifth maps described the later components P2p and P3, which were examined only to detail the sequence of processing events subsequent to the initial effects of spatial attention. In particular, the parietal P2 has been hypothesized to be concerned with the perception of contour and/or angularity of geometric figures (Brandeis \& Lehmann, 1989; Shoji \& Ozaki, 2006) and it was reported not to be modulated by selective attention (Talsma et al., 2005). In the present study a longer-latency P3, most likely a P3b, was distributed mainly over the centro-parietal regions of the right hemisphere and was more prominent with textures in comparison to arrows and eye-gaze. The P3b has been related to discrimination, categorization, matching and decision making (Desmedt, 1980; Mecklinger \& Ullsperger, 1993; Ruchkin, Johnson, 
Mahaffey, \& Sutton, 1988), confirming that textures required more cognitive resources than the other cues.

All in all, these effects suggest that arrows and eye-gaze induce the activation of the same cortical network as the endogenous texture cues. The occipito-parietal regions, which were the first to be involved in the processing of the visual information (P1), were probably modulated by top-down attentive influences related to the processing of specific visual features (N1). Concurrently, the orbitofrontal areas (P2a) were also involved. Given that participants took advantage from cueing already at the shortest SOAs and considering the interaction between endogenous and exogenous orienting, the electrophysiological correlates of the attentional shift induced by arrows and eye-gaze was searched in the early $200 \mathrm{~ms}$ after cue presentation. With respect to this, the EDAN effect was expected to provide relevant information. An EDAN-like effect was found with arrows and eye-gaze, but not with textures and earlier than reported in literature. At first sight, this result might indicate that an earlier attentional orienting was induced by arrows and eye-gaze in comparison to textures. Actually, this effect was present only with arrow cues, while with eye-gaze cues it showed an inverse pattern: rightward eye-gaze induced a smaller negativity in the left posterior hemisphere with respect to leftward cues. Doubts about the reliability of EDAN as ERP marker of attentional orienting have been already raised by Van Velzen and Eimer (2003). In keeping with that, in the present experiment the EDAN-like effect is most likely to reflect detection and selection mechanisms of taskrelevant features of attentional cue stimuli instead of attentional orienting. Arrows and eye-gaze differed systematically with respect to the relation between physical features and attentional direction. In both there were high-luminance (white) regions which were located ipsilaterally in arrows and contralaterally in eye-gaze relative to the cued direction. No high-luminance region was present in textures, accounting for the absence of the EDAN effect in this condition.

On the whole, these results are consistent with growing evidence that eye-gaze and arrows, as well as other socially relevant stimuli, mediate a very fast endogenous orienting of attention based on overlearned mechanisms (Hommel et al., 2001; Itier, Villate, \& Ryan, 2007; Vecera \& Rizzo, 2004, 2006). In daily life these stimuli assume an effective directional meaning able to influence other people's attention. Consequently, with practise the processing of their meaning becomes automatic and directly affects the spatial allocation of visual attention.

Hommel et al. (2001) showed that conventional, overlearned communicative signals can direct the visual attention of human observers in a relatively automatic fashion. They reported that arrows and directional words (right, left) facilitated the processing of stimuli appearing in locations matching with their meaning, although subjects were informed that they were non-predictive of target appearance. However, although this study demonstrated that this automatic-like orienting of attention concerns many socially relevant stimuli, it did not prove the non-reflexive nature of this mechanism. This possibility was investigated by Vecera and Rizzo (2006) by testing a patient with frontal lobe damage. They used three types of cues: eye-gaze, words (left, right) and peripheral flashes. Since the patient did not appear to orient attention to symbolic cues, but demonstrated the standard cueing effect with peripheral cues, the authors concluded that orienting to symbolic cues is an endogenous mechanism similar for all relevant social stimuli, but different from the reflexive orienting to peripheral targets subserved by subcortical areas (Klein, 2000; Sereno, Briand, Amador, \& Szapiel, 2006). Moreover, they suggested that the biological basis of this overlearned association mechanism are in the orbitofrontal cortex, which receives input from higher-order perceptual cortex and has access to formed perceptual represen- tations. Consequently, they are responsible both for learning and for recovering learned associations (Rolls, 1999). This "association hypothesis" in eye-gaze is supported also by a recent study of Itier et al. (2007), who investigated gaze-orienting behaviour by monitoring eye movements. Subjects were asked to perform two tasks: a gaze direction and a head orientation judgment. Results showed that although the eye region was the most attended feature in both tasks, the gaze-orienting behaviour was modulated by task demands, suggesting that orienting to gaze direction is not a reflexive mechanism.

In the present experiment two lines of evidence are consistent with these previous findings: first, the same network was engaged with all the symbolic cues; second, the involvement of the orbitofrontal cortex was probably reflected by the frontal P2a component, as suggested by previous sources studies (Potts et al., 2004). Its engagement in arrow and eye-gaze cues would produce the retrieval of the overlearned associations, while in texture condition it would be responsible for the learning of the new texture-direction association. The P2a component showed an earlier latency in eye-gaze in comparison to arrows and textures, as if the retrieval of the eye-gaze association was faster than the others. Given the lack of behavioural differences between eye-gaze and arrows, it is difficult to account for this ERP incongruency. However, eye-gaze undoubtedly represents a special stimulus with a great social and biological relevance. There are numerous previous suggestions for the innateness of gaze cognition: infants stare longer at the eyes than at other facial features (Maurer, 1985) and spontaneously follow someone else's gaze as early as 10 weeks of age (Hood, Willen, \& Driver, 1998). Moreover, the literature offers strong evidence, both through single-cell studies in monkeys (Desimone, 1991; Gross, 1992; Perrett, Mistlin, \& Chitty, 1987; Perrett et al., 1990) and electrophysiological (Allison, Puce, Spencer, \& McCarthy, 1999, 2000; Puce, Allison, Bentin, Gore, \& McCarthy, 1998; Puce \& Perrett, 2003) or functional imaging studies in humans (Hoffman \& Haxby, 2000; Hooker et al., 2003; Wicker, Michel, Henaff, \& Decety, 1998), that a specific region of the temporal lobe, such as the superior temporal sulcus, is involved in gaze processing. In the present study we did not find any ERP component or topography specific for the eye-gaze condition. The electrophysiological correlate of eyegaze, and more broadly of face encoding, is a negative wave (N170) peaking at $150-180 \mathrm{~ms}$ at posterior temporal sites (Bentin, Allison, Puce, Perez, \& McCarthy, 1996). The absence of N170 in the present experiment could be due to methodological differences regarding the reference used (see Joyce \& Rossion, 2005). Alternatively, it could depend on the absence of facial features except for schematic eyes (but see Taylor, Itier, Allison, \& Edmonds, 2001). It is also worth mentioning that a recent study raised doubts that N170 is a genuine ERP correlate of face processing (Thierry, Martin, Downing, \& Pegna, 2007).

However, even if gaze processing is subtended by specific neuronal mechanisms different from those involved in arrows processing, it is entirely possible that the subsequent orienting of attention is based on the same neuronal network. Previous neuroimaging studies, aiming at investigating the neural systems supporting the attentional orienting induced by arrows and eye-gaze, did not convincingly disentangle perception from attentional orienting (Hietanen, Nummenmaa, Nyman, Parkkola, \& Hamalainen, 2006; Kingstone, Tipper, Ristic, \& Ngan, 2004). Because of its poor temporal resolution, functional magnetic resonance imaging does not allow to investigate the time-course of fast cognitive responses. Kingstone et al. (2004) studied brain activation during an attention orienting task by using an ambiguous figure that could be perceived as eye-gaze or as a car. Both the perceptual conditions produced equivalent reflexive shifts of attention to the cued location, but the superior temporal sulcus was uniquely 
engaged when the stimulus was perceived as eyes. This result was used to claim that the neural systems subserving the two forms of orienting were not equivalent, but it was not mentioned at which stage of the processing difference emerged. By comparing directly the effects of gaze-cued and arrow-cued orienting, Hietanen et al. (2006) found two partially segregated cortical networks and this was interpreted as evidence that attention orienting by gaze and by arrow cues are not supported by the same cortical network and that the latter relies on mechanisms associated with voluntary shift of attention. Although neutral trials (i.e., a straight gaze and a segment line without arrows for eye-gaze and arrows, respectively) were used as control conditions in order to identify the neural mechanisms subserving the attentional orienting only (but see Senju \& Hasegawa, 2005), results still lacked the temporal information necessary to discriminate between stimulus processing and attentional orienting. To overcome this limitation, the same experiment was run by recording ERPs (Hietanen et al., 2008). The main finding was the presence of the EDAN effect with arrows but not with eye-gaze cues. The authors explained this result with the greater dependence of gaze cueing on subcortical mechanisms, whose activity is not detected by the ERP measures. Also in the present study an EDAN-like effect was present for arrows only, but its inverse pattern with eye-gaze and its lack with texture cues argue for a link with detection and selection more than with attentional orienting mechanisms. The two cueing conditions studied by Hietanen et al. (2008) seem to induce the same ERP components, but a systematic analysis of the topography was not applied. Therefore the amplitude differences observed between conditions are not convincing in proving the involvement of different attentional networks. However, given the different results of our experiment compared to that by Hietanen et al. (2008) more future investigations are required.

To conclude, the present study provides clear evidence that the attentional shift induced by eye-gaze and arrow cues is subserved by the same neural mechanisms, which appear to be identical to those engaged by a purely endogenous cue. Arrows and eye-gaze induced a very early attentional shift, but the similar pattern of ERP responses and its topography in the various conditions suggest that this early orienting of attention is more likely related to an overlearned association mechanism rather than to a pure exogenous attentional process. To provide decisive evidence supporting this hypothesis, future studies should ideally compare ERPs elicited by non-predicitve eye-gaze and arrows with those elicited by pure exogenous cues, such as peripheral cues, thereby testing the involvement of distinct neural mechanisms. However, at the moment this remains an intriguing challenge, since this issue of a comparison is complicated both at behavioural and electrophysiological level by the difficulty in balancing the attentional qualitative effects and the visual stimulation produced by central and peripheral cues.

\section{Acknowledgment}

Data were analysed using "Cartool" (http://brainmapping. unige.ch/cartool.php, by D. Brunet, Centre for Biomedical Imaging of Geneva and Lausanne, Switzerland).

\section{References}

AEEGS. (1991). American Electroencephalographic Society guidelines for standard electrode position nomenclature. Journal of Clinical Neurophysiology, 8, 200-202.

Allison, T., Puce, A., \& McCarthy, G. (2000). Social perception from visual cues: Role of the STS region. Trends in Cognitive Sciences, 4, 267-278.

Allison, T., Puce, A., Spencer, D. D., \& McCarthy, G. (1999). Electrophysiological studies of human face perception. I. Potentials generated in occipitotemporal cortex by face and non-face stimuli. Cerebral Cortex, 9, 415-430.
Anllo-Vento, L., Luck, S. J., \& Hillyard, S. A. (1998). Spatio-temporal dynamics of attention to color: Evidence from human electrophysiology. Human Brain Mapping, 6, 216-238.

Arnott, S. R., Pratt, J., Shore, D. I., \& Alain, C. (2001). Attentional set modulates visual areas: An event-related potential study of attentional capture. Brain Research. Cognitive Brain Research, 12, 383-395.

Bentin, S., Allison, T., Puce, A., Perez, E., \& McCarthy, G. (1996). Electrophysiological studies of face perception in humans. Journal of Cognitive Neuroscience, 8 , 551-565.

Brandeis, D., \& Lehmann, D. (1989). Segments of event-related potential map series reveal landscape changes with visual attention and subjective contours. Electroencephalography and Clinical Neurophysiology, 73, 507-519.

Cheal, M., \& Chastain, G. (2002). Efficiency of visual selective attention is related to the type of target. Psychological Research, 66, 110-115.

Clark, V.P., \& Hillyard, S. A. (1996). Spatial selective attention affects early extrastriate but not striate components of the visual evoked potential. Journal of Cognitive Neuroscience, 8, 387-402.

Corbetta, M., \& Shulman, G. L. (2002). Control of goal-directed and stimulus-driven attention in the brain. Nature Reviews. Neuroscience, 3, 201-215.

Corbetta, M., Miezin, F. M., Shulman, G. L., \& Petersen, S. E. (1993). A PET study of visuospatial attention. The Journal of Neuroscience, 13, 1202-1226.

Desimone, R. (1991). Face-selective cells in the temporal cortex of monkeys. Journal of Cognitive Neuroscience, 3, 1-8.

Desmedt, J. E. (1980). P300 in serial tasks: An essential post-decision closure mechanism. Progress in Brain Research, 54, 682-686.

Driver, J., \& Frackowiak, R. S. (2001). Neurobiological measures of human selective attention. Neuropsychologia, 39, 1257-1262.

Driver, J., Davis, G., Ricciardelli, P., Kidd, P., Maxwell, E., \& Baron-Cohen, S. (1999). Gaze perception triggers visuospatial orienting by adults in a reflexive manner. Visual Cognition, 6, 509-540.

Eason, R., Harter, M., \& White, C. (1969). Effects of attention and arousal on visually evoked cortical potentials and reaction time in man. Physiology and Behavior, 4, 283-289.

Eimer, M., \& Van Velzen, J. (2002). Crossmodal links in spatial attention are mediated by supramodal control processes: Evidence from event-related potentials. Psychophysiology, 39, 437-449.

Eimer, M., \& Kiss, M. (2008). Involuntary attentional capture is determined by task set: Evidence from event-related brain potentials. Journal of Cognitive Neuroscience,

Eimer, M., van Velzen, J., \& Driver, J. (2002). Cross-modal interactions between audition, touch, and vision in endogenous spatial attention: ERP evidence on preparatory states and sensory modulations. Journal of Cognitive Neuroscience, $14,254-271$.

Folk, C. L., Remington, R. W., \& Johnston, J. C. (1992). Involuntary covert orienting is contingent on attentional control settings. Journal of Experimental Psychology. Human Perception and Performance, 18, 1030-1044.

Friesen, C. K., \& Kingstone, A. (1998). The eyes have it! Reflexive orienting is triggered by nonpredictive gaze. Psychonomic Bulletin \& Review, 5, 490-495.

Friesen, C. K., Ristic, J., \& Kingstone, A. (2004). Attentional effects of counterpredictive gaze and arrow cues. Journal of Experimental Psychology. Human Perception and Performance, 30, 319-329.

Gabay, S., \& Henik, A. (2008). The effects of expectancy on inhibition of return. Cognition, 106, 1478-1486.

Gibson, B. S., \& Bryant, T. A. (2005). Variation in cue duration reveals top-down modulation of involuntary orienting to uninformative symbolic cues. Perception E Psychophysics, 67, 749-758.

Gomez Gonzales, C. M., Clark, V. P., Fan, S., Luck, S. J., \& Hillyard, S. A. (1994). Sources of attention-sensitive visual event-related potentials. Brain Topography, 7 , 41-51.

Gross, C. G. (1992). Representation of visual stimuli in inferior temporal cortex. Philosophical Transactions of the Royal Society of London. Series B, Biological Sciences, 335, 3-10.

Harter, M. R., Aine, C., \& Schroeder, C. (1982). Hemispheric differences in the neural processing of stimulus location and type: Effects of selective attention on visual evoked potentials. Neuropsychologia, 20, 421-438.

Harter, M. R., Miller, S. L., Price, N. J., LaLonde, M. E., \& Keyes, A. L. (1989). Neural processes involved in directing attention. Journal of Cognitive Neuroscience, 1, 223-237.

Hickey, C., McDonald, J. J., \& Theeuwes, J. (2006). Electrophysiological evidence of the capture of visual attention. Journal of Cognitive Neuroscience, 18, 604-613.

Hietanen, J. K., Leppanen, J. M., Nummenmaa, L., \& Astikainen, P. (2008). Visuospatial attention shifts by gaze and arrow cues: An ERP study. Brain Research, 1215, 123-136.

Hietanen, J. K., Nummenmaa, L., Nyman, M. J., Parkkola, R., \& Hamalainen, H. (2006). Automatic attention orienting by social and symbolic cues activates different neural networks: An fMRI study. Neuroimage, 33, 406-413.

Hillyard, S. A., \& Picton, T. W. (1979). Event-related brain potentials and selective information processing in man. In J. E. Desmedt (Ed.), Progress in clinical neurophysiology cognitive components in cerebral event-related potentials and selective attention (pp. 1-50). Basel: Karger.

Hoffman, E. A., \& Haxby, J. V. (2000). Distinct representations of eye gaze and identity in the distributed human neural system for face perception. Nature Neuroscience, 3, 80-84.

Hommel, B., Pratt, J., Colzato, L., \& Godijn, R. (2001). Symbolic control of visual attention. Psychological Science, 12, 360-365. 
Hood, B. M., Willen, J. D., \& Driver, J. (1998). Adult's eyes trigger shifts of visual attention in human infants. Psychological Science, 9, 53-56.

Hooker, C. I., Paller, K. A., Gitelman, D. R., Parrish, T. B., Mesulam, M. M., \& Reber, P. J. (2003). Brain networks for analyzing eye gaze. Brain Research. Cognitive Brain Research, 17, 406-418.

Hopf, J. M., \& Mangun, G. R. (2000). Shifting visual attention in space: An electrophysiological analysis using high spatial resolution mapping. Clinical Neurophysiology, 111, 1241-1257.

Hopfinger, J. B., \& Mangun, G. R. (1998). Reflexive attention modulates processing of visual stimuli in human extrastriate cortex. Psychologiacal Science, 9, 441-447.

Hopfinger, J. B., \& Mangun, G. R. (2001). Tracking the influence of reflexive attention on sensory and cognitive processing. Cognitive, Affective \& Behavioral Neuroscience, 1, 56-65.

Itier, R. J., \& Taylor, M. J. (2004). N170 or N1? Spatiotemporal differences between object and face processing using ERPs. Cerebral Cortex, 14, 132-142.

Itier, R. J., Villate, C., \& Ryan, J. D. (2007). Eyes always attract attention but gaze orienting is task-dependent: Evidence from eye movement monitoring. Neuropsychologia, 45, 1019-1028.

Jonides, J. (1981). Voluntary vs. automatic control over the mind's eye's movement. In J. B. Long \& A. D. Baddeley (Eds.), Attention and performance. IX. Hillsdale, NJ: Lawrence Erlbaum Associates.

Jonides, J., \& Yantis, S. (1988). Uniqueness of abrupt visual onset in capturing attention. Perception \& Psychophysics, 43, 346-354.

Joyce, C., \& Rossion, B. (2005). The face-sensitive N170 and VPP components manifest the same brain processes: The effect of reference electrode site. Clinical Neurophysiology, 116, 2613-2631.

Kenemans, J. L., Kok, A., \& Smulders, F. T. (1993). Event-related potentials to conjunctions of spatial frequency and orientation as a function of stimulus parameters and response requirements. Electroencephalography and Clinical Neurophysiology, 88, 51-63.

Kim, Y. H., Gitelman, D. R., Nobre, A. C., Parrish, T. B., LaBar, K. S., \& Mesulam, M. M. (1999). The large-scale neural network for spatial attention displays multifunctional overlap but differential asymmetry. Neuroimage, 9, 269-277.

Kingstone, A., Tipper, C., Ristic, J., \& Ngan, E. (2004). The eyes have it!: An fMR investigation. Brain and Cognition, 55, 269-271.

Klein, R. M. (2000). Inhibition of return. Trends in Cognitive Science, 4, 138-147.

Lambert, A., Spencer, E., \& Mohindra, N. (1987). Automaticity and the capture of attention by a peripheral display change. Current Psychological Research Reviews, $6,136-147$.

Langton, S. R. H., \& Bruce, V. (1999). Reflexive social orienting. Visual Cognition, 6, 541-567.

Leber, A. B., \& Egeth, H. E. (2006). It's under control: Top-down search strategies can override attentional capture. Psychonomic Bulletin E' Review, 13, 132-138.

Lehmann, D., \& Skrandies, W. (1980). Reference-free identification of components of checkerboard-evoked multichannel potential fields. Electroencephalography and Clinical Neurophysiology, 48, 609-621.

Luck, S. J., Heinze, H. J., Mangun, G. R., \& Hillyard, S. A. (1990). Visual event-related potentials index focused attention within bilateral stimulus arrays. II. Functional dissociation of P1 and N1 components. Electroencephalography and Clinical Neurophysiology, 75, 528-542.

Luck, S. J., Hillyard, S. A., Mouloua, M., Woldorff, M. G., Clark, V. P., \& Hawkins, H. L. (1994). Effects of spatial cuing on luminance detectability: Psychophysical and electrophysiological evidence for early selection. Journal of Experimental Psychology. Human Perception and Performance, 20, 887-904.

Makeig, S., Westerfield, M., Jung, T. P., Covington, J., Townsend, J., Sejnowski, T. J., \& Courchesne, E. (1999). Functionally independent components of the late positive event-related potential during visual spatial attention. The Journal of Neuroscience, 19, 2665-2680.

Mangun, G. R., \& Hillyard, S. A. (1991). Modulations of sensory-evoked brain potentials indicate changes in perceptual processing during visual-spatial priming. Journal of Experimental Psychology. Human Perception and Performance, 17, 1057-1074.

Mangun, G. R., Hillyard, S. A., \& Luck, S. J. (1993). Electrocortical substrates of visual selective attention. In D. Meyer \& S. Kornblum (Eds.), Attention and performance. XIV. synergies in experimental psychology, artificial intelligence and cognitive neuroscience (pp. 219-243). Cambridge, MA: MIT Press.

Maurer, D. (1985). T. Field \& N. Fox (Eds.), Infants' perception of facedness. Social perception in infants Ablex: Norwood, NJ., pp. 73-100.

Mecklinger, A., \& Ullsperger, P. (1993). P3 varies with stimulus categorization rather than probability. Electroencephalography and Clinical Neurophysiology, 86 395-407.

Michel, C. M., Seeck, M. \& Landis, T. (1999). Spatiotemporal dynamics of human cognition. News in Physiological Sciences, 14, 206-214.

Milliken, B., Lupianez, J., Roberts, M., \& Stevanovski, B. (2003). Orienting in space and time: Joint contributions to exogenous spatial cuing effects. Psychonomic Bulletin \& Review, 10, 877-883.

Muller, H. J., \& Findlay, J. M. (1988). The effect of visual attention on peripheral discrimination thresholds in single and multiple element displays. Acta Psychologica (Amsterdam), 69, 129-155.

Muller, H. J., \& Rabbitt, P. M. (1989). Reflexive and voluntary orienting of visual attention: Time course of activation and resistance to interruption Journal of Experimental Psychology. Human Perception and Performance, 15, 315330.

Murray, M. M., Brunet, D., \& Michel, C. M. (2008). Topographic ERP analyses: A stepby-step tutorial review. Brain Topography, 20, 249-264.
Näätänen, R. (1975). Selective attention and evoked potentials in humans-a critical review. Biological Psychology, 2, 237-307.

Nobre, A. C., Sebestyen, G. N., \& Miniussi, C. (2000). The dynamics of shifting visuospatial attention revealed by event-related potentials. Neuropsychologia, 38 964-974.

Nobre, A. C., Sebestyen, G. N. Gitelman, D. R., Mesulam, M. M., Frackowiak, R. S., \& Frith, C. D. (1997). Functional localization of the system for visuospatial attention using positron emission tomography. Brain, 120(Pt 3), 515-533.

Oldfield, R. C. (1971). The assessment and analysis of handedness: The Edinburgh inventory. Neuropsychologia, 9, 97-113.

Pascual-Marqui, R. D., Michel, C. M., \& Lehmann, D. (1995). Segmentation of brain electrical activity into microstates: Model estimation and validation. IEEE Transactions on Biomedical Engineering, 42, 658-665.

Peelen, M. V., Heslenfeld, D. J., \& Theeuwes, J. (2004). Endogenous and exogenous attention shifts are mediated by the same large-scale neural network. Neuroimage, 22, 822-830.

Perrett, D. I., Mistlin, A. J., \& Chitty, A. J. (1987). Visual neurones responsive to faces. Trends in Neuroscience, 10, 358-364.

Perrett, D. I., Harries, M. H., Mistlin, A. J., Hietanen, J. K., Benson, P. J., Bevan, R., Thomas, S., Oram, M. W., \& Brierly, K. (1990). Social signals analyzed at the cell level: Someone is looking at me, something touched me, something moved! International Journal of Comparative Psychology, 4.

Posner, M. I. (1980). Orienting of attention. The Quarterly Journal of Experimental Psychology, 32, 3-25.

Potts, G. F. (2004). An ERP index of task relevance evaluation of visual stimuli. Brain Cognition, 56, 5-13.

Potts, G. F., \& Tucker, D. M. (2001). Frontal evaluation and posterior representation in target detection. Brain Research. Cognitive Brain Research, 11, 147-156.

Potts, G. F., Patel, S. H., \& Azzam, P. N. (2004). Impact of instructed relevance on the visual ERP. International Journal of Psychophysiology, 52, 197-209.

Potts, G. F., Liotti, M., Tucker, D. M., \& Posner, M. (1996). Frontal and inferior tempora cortical activity in visual target detection: Evidence from high spatially sampled event-related potentials. Brain Topography, 9, 3-14.

Pourtois, G., De Pretto, M. Hauert, C. A., \& Vuilleumier, P. (2006). Time course of brain activity during change blindness and change awareness: Performance is predicted by neural events before change onset.Journal of Cognitive Neuroscience. $18,2108-2129$.

Pourtois, G., Dan, E. S., Grandjean, D., Sander, D., \& Vuilleumier, P. (2005). Enhanced extrastriate visual response to bandpass spatial frequency filtered fearful faces: Time course and topographic evoked-potentials mapping. Human Brain Mapping, 26, 65-79.

Puce, A., \& Perrett, D. (2003). Electrophysiology and brain imaging of biological motion. Philosophical Transactions of the Royal Society of London. Series B, Biological Sciences, 358, 435-445.

Puce, A., Allison, T., Bentin, S., Gore, J. C., \& McCarthy, G. (1998). Temporal cortex activation in humans viewing eye and mouth movements. The Journal of Neuroscience, 18, 2188-2199.

Ristic, J., Friesen, C. K., \& Kingstone, A. (2002). Are eyes special? It depends on how you look at it. Psychonomic Bulletin \& Review, 9, 507-513.

Rolls, E. T. (1999). The functions of the orbitofrontal cortex. Neurocase, 5, 301-312.

Rosen, A. C., Rao, S. M., Caffarra, P., Scaglioni, A., Bobholz, J. A., Woodley, S. J., Hammeke, T. A., Cunningham, J. M., Prieto, T. E., \& Binder, J. R. (1999). Neural basis of endogenous and exogenous spatial orienting. A functional MRI study. Journal of Cognitive Neuroscience, 11, 135-152.

Ruchkin, D. S., Johnson, R., Jr., Mahaffey, D., \& Sutton, S. (1988). Toward a functional categorization of slow waves. Psychophysiology, 25, 339-353.

Rugg, M., \& Coles, M. (1995). Electrophysiology of mind: event-related brain potentials and cognition. Oxford, England: Oxford University Press.

Ruz, M., \& Nobre, A. C. (2008). Dissociable top-down anticipatory neural states for different linguistic dimensions. Neuropsychologia, 46, 1151-1160.

Schuller, A. M., \& Rossion, B. (2001). Spatial attention triggered by eye gaze increases and speeds up early visual activity. Neuroreport, 12, 23812386.

Schuller, A. M., \& Rossion, B. (2004). Perception of static eye gaze direction facilitates subsequent early visual processing. Clinical Neurophysiology, 115, 1161-1168.

Senju, A., \& Hasegawa, T. (2005). Direct gaze captures visuospatial attention. Visua Cognition, 12, 127-144

Sereno, A. B., Briand, K. A., Amador, S. C., \& Szapiel, S. V. (2006). Disruption of reflexive attention and eye movements in an individual with a collicular lesion. Journal of Clinical and Experimental Neuropsychology, 28, 145-166.

Shoji, H., \& Ozaki, H. (2006). Topographic change in ERP due to discrimination of geometric figures in the peripheral visual field. International Journal of Psychophysiology, 62, 115-121.

Smid, H. G., Jakob, A., \& Heinze, H. J. (1999). An event-related brain potential study of visual selective attention to conjunctions of color and shape. Psychophysiology, 36, 264-279.

Talsma, D., Slagter, H. A., Nieuwenhuis, S., Hage, J., \& Kok, A. (2005). The orienting of visuospatial attention: An event-related brain potential study. Brain Research. Cognitive Brain Research, 25, 117-129.

Taylor, M. J., Itier, R. J., Allison, T., \& Edmonds, G. E. (2001). Direction of gaze effects on early face processing: Eyes-only versus full faces. Brain Research. Cognitive Brain Research, 10, 333-340.

Thierry, G., Martin, C. D., Downing, P., \& Pegna, A. J. (2007). Controlling for interstimulus perceptual variance abolishes N170 face selectivity. Nature Neuroscience, 10 505-511. 
Tipples, J. (2002). Eye gaze is not unique: Automatic orienting in response to uninformative arrows. Psychonomic Bulletin \& Review, 9, 314-318.

Van Velzen, J., \& Eimer, M. (2003). Early posterior ERP components do not reflect the control of attentional shifts toward expected peripheral events. Psychophysiology, 40, 827-831.

Vecera, S. P., \& Rizzo, M. (2004). What are you looking at? Impaired 'social attention' following frontal-lobe damage. Neuropsychologia, 42, 1657-1665.

Vecera, S. P., \& Rizzo, M. (2006). Eye gaze does not produce reflexive shifts of attention: Evidence from frontal-lobe damage. Neuropsychologia, 44, 150159.

Vogel, E. K., \& Luck, S. J. (2000). The visual N1 component as an index of a discrimination process. Psychophysiology, 37, 190-203.
Warner, C. B., Juola, J. F., \& Koshino, H. (1990). Voluntary allocation versus automatic capture of visual attention. Perception E Psychophysics, 48, 243-251.

Wicker, B., Michel, F., Henaff, M. A., \& Decety, J. (1998). Brain regions involved in the perception of gaze: A PET study. Neuroimage, 8, 221-227.

Wijers, A. A., Mulder, G., Okita, T., \& Mulder, L. J. (1989). An ERP study on memory search and selective attention to letter size and conjunctions of letter size and color. Psychophysiology, 26, 529-547.

Yamaguchi, S., Tsuchiya, H., \& Kobayashi, S. (1994). Electroencephalographic activity associated with shifts of visuospatial attention. Brain, 117(Pt 3), 553-562.

Yantis, S., \& Jonides, J. (1984). Abrupt visual onsets and selective attention: Evidence from visual search. Journal of Experimental Psychology. Human Perception and Performance, 10, 601-621. 